\title{
Pediatric Anesthesiology Review, 2nd Edition
}

\author{
Robert S. Holzman, Thomas J. Mancuso, Joseph P. Cravero, James A. DiNardo. Springer, \\ Switzerland, 2017. \$199.00 (paperback version). 778 pages. ISBN 978-3-319-48447-1
}

\author{
Christina Staniforth, MD, FRCPC
}

Received: 12 January 2018/Revised: 18 January 2018/Accepted: 19 January 2018/Published online: 15 March 2018

(c) Canadian Anesthesiologists' Society 2018

Pediatric Anesthesiology Review is the second edition of a self-assessment text originally published in 2011. As stated in the title, it is a focused review book, not an expansively referenced text. The editors are anesthesiologists based in Boston, Massachusetts at Boston Children's Hospital and/ or Harvard Medical School. They have collected this vast amount of knowledge specifically for residents and fellows with the goal of enhancing their education on their way to becoming consultants in pediatric anesthesia.

The Review is organized into two sections, the first of which focuses on, and is entitled, "Pediatric Medicine for Pediatric Anesthesiologists." The second, larger portion is devoted to consultation medicine, appropriately labelled "Consultations in Pediatric Anesthesia." The layout of each section is meant to encourage emergence of the reader's own thoughts (and self-study) and to facilitate independent establishment of ideas via probing questions, thereby gaining more in-depth understanding of the particular topic.

This goal is accomplished differently in each of the two sections. The first is organized into approximately 130 pages of multiple-choice style questions on the left-hand side of the page with the corresponding answers on the right-hand side. There are seven chapters in this format including titles such as "Newborn Medicine," "The Respiratory System," and "Cardiology." The responses not only state the correct answer(s) but provide an explanation along with a few important facts about the chosen topic. The purpose of this segment of the book, with

C. Staniforth, MD, FRCPC ( $₫)$

Departments of Anesthesia and Pediatric Anesthesia, University of Manitoba, Winnipeg, MB, Canada

e-mail: umkolt@myumanitoba.ca the most important areas well highlighted, is to ensure that the pediatric consultant has excellent baseline knowledge of pediatric medicine. References and/or suggested readings are included at the end of most of the chapters, but individual questions are not specifically referenced, often forcing readers to search elsewhere for references if they have questions.

The second portion - the bulk of the textbook (i.e., $600+$ pages) - focuses on specific subspecialty areas of pediatric anesthesia. Each chapter begins by presenting a case, followed by a series of specific questions about it. Similar to the first half of the book, questions are posed on the left-hand side with their corresponding responses on the right. Black and white illustrations are often used to highlight important aspects of the text. They are used appropriately, although not frequently. Following completion of the main scenario, additional areas of importance in each subject area are addressed. Chapter subjects range from expected pediatric topics such as "Newborn Emergencies" and "Head and Neck" surgeries, to more specialized areas such as "Conjoined Twins." Although each topic may not be systematically covered as it would be in a larger, referenced textbook, Review carefully highlights not-to-be-missed areas. It engages the learner with constant questioning while expecting the reader to already possess some knowledge of pediatric anesthesia.

Overall, Pediatric Anesthesiology Review, 2nd Edition is a good supplemental text for a resident or fellow looking to gain additional practical knowledge in this field. It is an easy read whether one starts at the opening chapter and follows each subject in the presented sequence or simply opens it to a chapter of particular interest. 
The text does have some limitations. For example, readers must keep in mind that they may need to look elsewhere for reference material if they have further questions. Also, locating references for many of the responses may prove difficult as they are not cited adjacent to the answers, and some of the references at the end of the chapters either cannot be identified as referring to specific questions or are incomplete. Another problem is that the Review's question/answer format may not be a suitable method of learning for all readers. Finally, there are occasional minor editing errors in this second edition. For example, in the multiple-choice section, where an answer of "D" is given as correct, the text states that "multiple" answers are correct. Errors such as this would have been noticeable even to someone with limited knowledge of pediatric anesthesia.

Despite a few shortcomings, I recommend Pediatric Anesthesiology Review to anyone looking for information about pediatric anesthesia beyond what appears in standard, referenced textbooks. It will also appeal to the reader who is looking for an engaging book to aid their independent quest to become a consultant in pediatric anesthesia.

Conflicts of interest None declared.

Editorial responsibility This submission was handled by Dr. Hilary P. Grocott, Editor-in-Chief, Canadian Journal of Anesthesia. 